\title{
Specifying the Representation of Non-geometric Information in 3D Virtual Environments
}

\author{
Kaveh Bazargan and Gilles Falquet \\ CUI - University of Geneva \\ 7, route de Drize, $\mathrm{CH}-1227$ Carouge, Switzerland \\ \{kaveh.bazargan, gilles. falquet\}@unige.ch
}

\begin{abstract}
In 3D virtual environments (3DVE), we need to know what an object looks like (i.e. geometric information) and what the object is, what are its properties and characteristics and how it relates to other objects (i.e. non-geometric information). Several interactive presentation techniques have been devised to incorporate non-geometric information into 3DVEs. The relevance of a technique depends on the context. Therefore, the choice of an appropriate representation technique cannot be done once for all and must be adapted to the context. In this paper, we first present a preliminary classification of representation techniques for non-geometric information in 3DVE. Then we propose a formalism, based on description logics, to describe the usability of a technique in a given context. We show how these descriptions can be processed to select appropriate techniques when automatically or semi-automatically generating a 3DVE.
\end{abstract}

Keywords: Information-rich virtual environments, 3D interaction techniques, Human computer interaction, Usability.

\section{Introduction}

$3 \mathrm{D}$ virtual environments (3DVE) are 3D user interfaces that are intended to represent geometric objects, like buildings, roads, trees, engine parts, etc. In order to perform a task in 3DVE, a user generally needs more knowledge than what is represented by 3D objects. For instance, he or she needs to know not only what an object looks like, but also what the object is, what are its properties and characteristics and how an object relates to other objects. Therefore, in addition to 3D geometry, these applications must integrate non-geometric information in the virtual environment. These environments are frequently referred to as semantically enriched virtual environments. Many types of non-geometric information can appear in such environments: simple numeric values associated to 3D objects (e.g. the heat of a part); semantic relationships between objects (e.g. "this parking lot belongs to that building"); or abstract concepts (e.g. safety instructions for operating a device).

Several interactive presentation techniques have been devised to incorporate these types of information into 3DVE: Illustrative Shadows [1], Virtual PDA [2], Croquet 3D Windows [3] \& Interactors [4], Interactive 2D Media Layers [5], Sidebar [6], 3D labels [7] and many more. Obviously, none of them is intrinsically better than the 
others in all situations. Indeed, the suitability of a technique depends on several factors such as the user profile, the current task the user is performing, the location in the 3D environment, or the characteristics of the information to display (size, semantic links to other information and so on). Thus, the choice of an appropriate representation technique cannot be done once for all; it must be constantly adapted to the context. Our research is limited to the usability study of selected abstract information representation techniques in 3DVE dedicated to performing two main tasks: consulting information and visualising relations.

When considering user interface (UI) generation this means that the UI designer must be provided with adequate tools to specify which representation techniques to use in which context. UI designers should be able to specify the adaptation of the presentation, for each kind of non-geometric information. In order to obtain usable interfaces, with enhanced usability, the UI designer should also be provided with ergonomic guidelines, as design patterns, to help her or him to select appropriate representation techniques. In this paper, we propose a model and language to specify the adaptive choice of representation techniques.

\section{Interaction Techniques in 3D Virtual Environments}

Interaction techniques provide the user with means to execute different types of task in 3D space. Therefore, interaction techniques are classified according to the tasks that they support. Bowman [8] proposed three basic interaction tasks in 3D user interfaces: the object selection and the object manipulation to handle 3D objects inside the $3 \mathrm{D}$ scene and the navigation to explore the 3D scene.

Existing 3D interaction techniques are divided into two groups: SelectionManipulation and Navigation. 3D widgets [9] can be used to put controls on objects. Both perceptual information and abstract information are subjected to selection and manipulation. Examples of perceptual information are position, shape, color, texture etc. of $3 \mathrm{D}$ object. Abstract information is information that is not normally directly perceptible in the physical world. For example, information about the visual appearance or surface texture of a table is directly perceptible, while information about its date and place of manufacture is not (this information is thus abstract) [10]. Several selection and manipulation techniques have been created and several classifications [11] of interaction techniques have also been proposed.

Navigation in 3D environments is made of two components: the motor component and the decision-making component. In the literature, the motor component is called by different names such as motion, locomotion, travel or viewpoint motion control [8]. The decision-making component is usually known as wayfinding. The interaction techniques for navigation include travel techniques and way finding aids. Travel techniques support exploration and manoeuvring tasks while wayfinding aids are helpful for search tasks. Navigation is composed of exploration, search and manoeuvering tasks. Travel techniques such as physical movement, manual viewpoint manipulation, direction specification (steering), destination specification (target-based travel) and path specification (route planning) support the exploration and the manoeuvering tasks. Wayfinding aids such as map, artificial landmark [12] etc. support search tasks. 
Other ways of interaction are related with tasks that involve issuing commands to the application in order to change system mode or activate some functionality. Techniques that support system control tasks in three-dimensions are graphical menus, voice commands, gestural interaction and virtual tools [13] with specific functions. A survey of existing 3D menus along with a taxonomy considering their hierarchical nature is provided in [14].

\section{Representation Techniques for Non-geometric Information}

In this section we present and classify selected techniques that have been devised and used to represent non-geometric information in 3DVE. This classification is based on the following criteria:

Layout space and placement. Polys [15] has revised the design space originally proposed in [10] to more accurately capture the nuances regarding where the abstract information is located and how it is associated to the perceptual information. Five types of layout spaces have been identified. In the Object Space layout, abstract information is always linked to the object it describes, even if the object is moved. The World Space layout is relative to a fixed area, region, or location in the environment. The User Space layout is relative to the user's location but not their viewing angle. The Viewport Space layout is related to the superimposed image plane where overlays and layers are located. In the Display Space layout space abstract visualizations are located outside the rendered view in some additional screen area.

Type of media. Integrating rich media types in 3DVE improves how users perceive, understand, and interact with visual representations of abstract information. Each presentation technique is designed to handle a specific type of media or a specific information structure. This can range from mere numbers and strings to sets textual information, multimedia documents, tables or graph structures.

Size of media or information density. To achieve usable results for users to understand and interpret the 3DVE, each technique must ensure that a fragment of abstract information is visible and unambiguously associated with an object located in the 3D scene. For instance, in dense or crowded scenes with a large number of annotation panels, users can be quickly overwhelmed or confused as annotations consume the visual space. This visibility issue sets a number of constraints on the size of the media. For instance, if the media is text, the size can range from a short label composed of few words to several paragraphs.

Our review of related work shows that labeling of 3DVE are well studied, but, representation techniques for integrating abstract information in 3DVE have not yet been systematically classified and studied. Table 1 provides a selected list of interactive representation techniques for non-geometric information in 3DVE.

Illustrative Shadows. [1] are projections onto a flat plane that enables schematic illustrations which are focused on specific information extraction tasks and facilitates the integration of generated textual information that leads to further meaning. Illustrative Shadows provide an intuitive visual link and a level of integration between interactive $3 \mathrm{D}$ graphics and supplemental $2 \mathrm{D}$ information displays that is hard to achieve with other concepts (see Figure 1). 
Table 1. Selected list of interactive representation techniques for non-geometric information in 3DVE

\begin{tabular}{|l|l|l|l|l|l|}
\hline Attribute & $\begin{array}{l}\text { Layout } \\
\text { space }\end{array}$ & $\begin{array}{l}\text { Type of } \\
\text { media }\end{array}$ & Size of media & $\begin{array}{l}\text { Typical } \\
\text { application } \\
\text { domain }\end{array}$ & Ref. \\
\hline $\begin{array}{l}\text { Illustrative } \\
\text { Shadows }\end{array}$ & $\begin{array}{l}\text { object \& } \\
\text { viewport } \\
\text { space }\end{array}$ & $\begin{array}{l}\text { short text, } \\
\text { image }\end{array}$ & $\begin{array}{l}\text { 1 paragraph, } \\
\text { small image }\end{array}$ & $\begin{array}{l}\text { 3D medical } \\
\text { education \& } \\
\text { training }\end{array}$ & {$[1]$} \\
\hline $\begin{array}{l}\text { Virtual } \\
\text { PDA }\end{array}$ & $\begin{array}{l}\text { world } \\
\text { space }\end{array}$ & multimedia & any size & $\begin{array}{l}\text { 3D science } \\
\text { education }\end{array}$ & {$[2]$} \\
\hline $\begin{array}{l}\text { Croquet 3D } \\
\text { Windows }\end{array}$ & $\begin{array}{l}\text { user } \\
\text { space }\end{array}$ & multimedia & any size & $\begin{array}{l}\text { collaborative } \\
\text { 3D platform }\end{array}$ & {$[3]$} \\
\hline $\begin{array}{l}\text { Croquet 3D } \\
\text { Interactor }\end{array}$ & $\begin{array}{l}\text { object } \\
\text { space }\end{array}$ & $\begin{array}{l}\text { text label, } \\
\text { thumbnail }\end{array}$ & $\begin{array}{l}\text { few words, } \\
\text { small image }\end{array}$ & $\begin{array}{l}\text { collaborative } \\
\text { 3D platform }\end{array}$ & {$[4]$} \\
\hline $\begin{array}{l}\text { 2D Media } \\
\text { Layer }\end{array}$ & $\begin{array}{l}\text { viewport } \\
\text { space }\end{array}$ & multimedia & any size & $\begin{array}{l}\text { virtual museum } \\
\text { visit }\end{array}$ & {$[5]$} \\
\hline Sidebar & $\begin{array}{l}\text { display } \\
\text { space }\end{array}$ & $\begin{array}{l}\text { short text, list, } \\
\text { thumbnail }\end{array}$ & $\begin{array}{l}\text { few para- } \\
\text { graphs, } \\
\text { small image }\end{array}$ & $\begin{array}{l}\text { training } \\
\text { \& simulation }\end{array}$ & {$[6]$} \\
\hline 3D labels & $\begin{array}{l}\text { object } \\
\text { space }\end{array}$ & text label & few words & virtual city visit & {$[7]$} \\
\hline
\end{tabular}

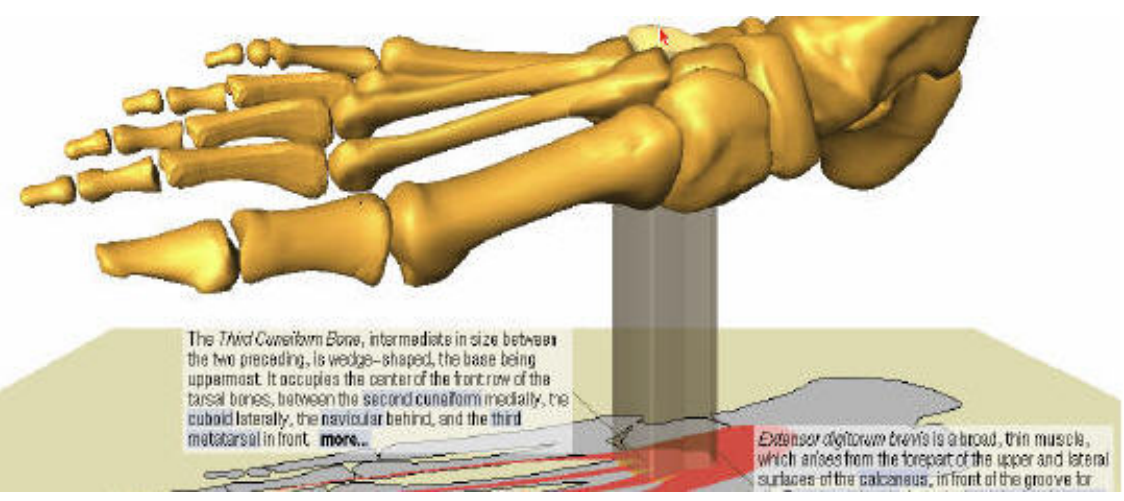

Fig. 1. Illustrative Shadows

Virtual PDAs. [2] are intended to display information not directly related to a particular object but more general information about the scene. For instance, one of the PDA's buttons can display a 2D map of the scene, while another button shows messages coming from an automated agent that sends suggestions to the scene visitor (see Figure 2).

Croquet 3D windows. [3] are general windows that live within the 3D scene. They can display any type of information: texts, graphs, spreadsheets, images, etc. They also serve as portals to jump to other worlds (scenes). In addition they are equipped with 3D 


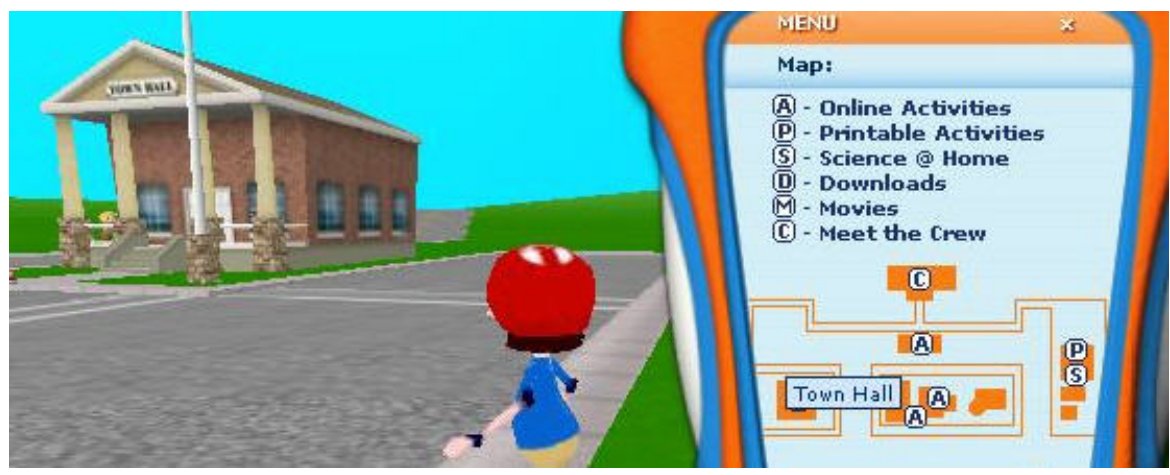

Fig. 2. 2D map displayed by a Virtual PDA

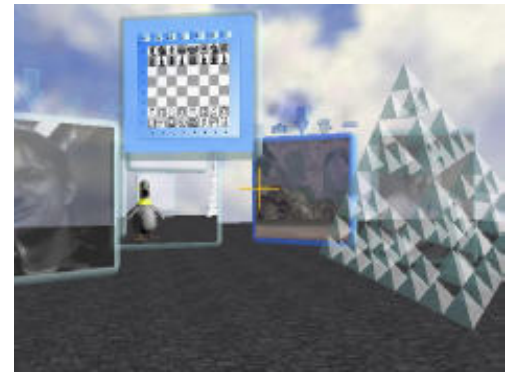

(a)

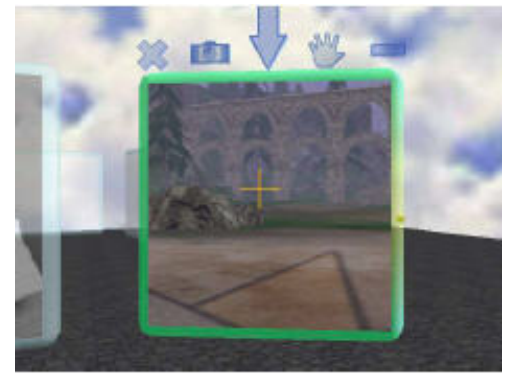

(b)

Fig. 3. Croquet 3D windows (a) and 3D buttons details (b)

buttons that sit on top of them. A particularly interesting feature is the downward arrow button that is intended to move the user from her current location to some place in front of the window, from where the window's content is easy to see. Thus, it saves the user the effort to walk to a suitable viewpoint (see Figure 3(a) and 3(b)).

Croquet 3D interactors [4] are similar to magic lenses that, when pointed at a particular object, reveal annotations attached to this object. Interactors act selectively, meaning that they reveal only annotations made through the same interactor. Thus an interactor is a kind of viewpoint related 3D generalization lens [16]. Interactors can be combined to simultaneously show annotations belonging to several viewpoints (see Figure 4).

The interactive 2D media layer technique [5] is a kind of 2D menu composed of elements in form of icons. When activated, by clicking, the 2D layer displays a panel, the elements of which are icons arranged in rows and columns. Clicking on an icon displays the related document (a image, a text, or a video). The $2 \mathrm{D}$ layer is not strongly connected to one scene object, but its location indicates that it contains information about the surrounding objects (see Figure 5). 


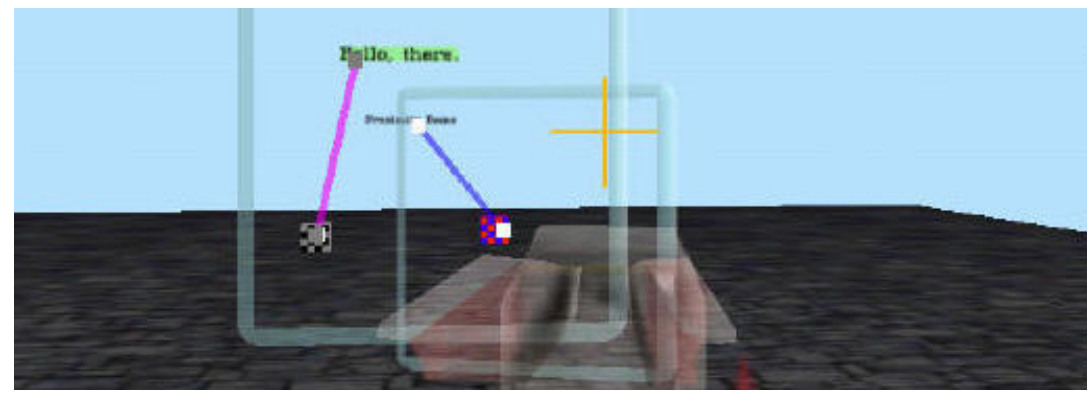

Fig. 4. Croquet 3D interactors

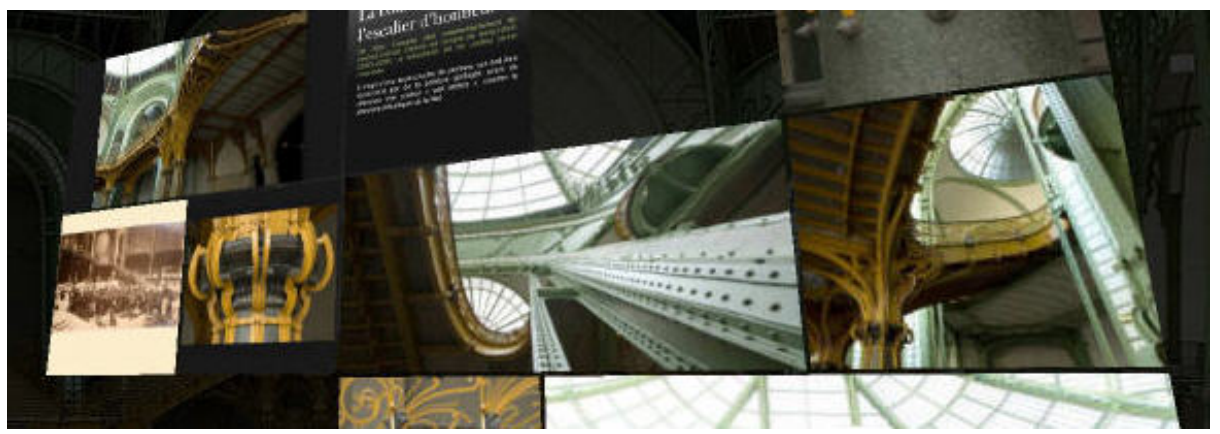

Fig. 5. Interactive 2D media layer

The sidebar technique [6] is designed to let the user interact with objects within the 3D scene and navigate from one component to another via textual description listed in a vertical sidebar menu (see Figure 6).

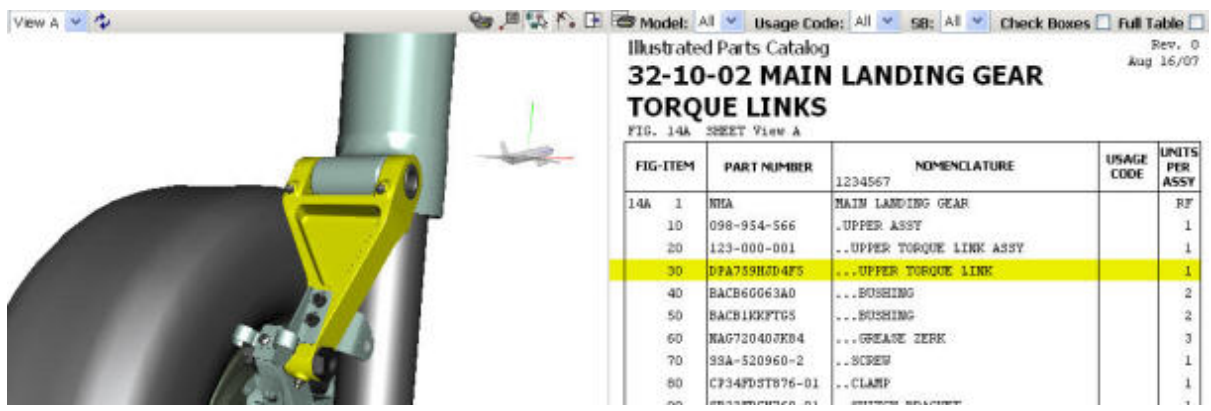

Fig. 6. Sidebar

The 3D labels technique [7] is based on the automated, dynamic placement of labels attached to objects of 3D scenes. These labels are seamlessly integrated into the 3D scene by textured polygons aligned to parameterized hulls, which generalize an object's geometry (see Figure 7). 


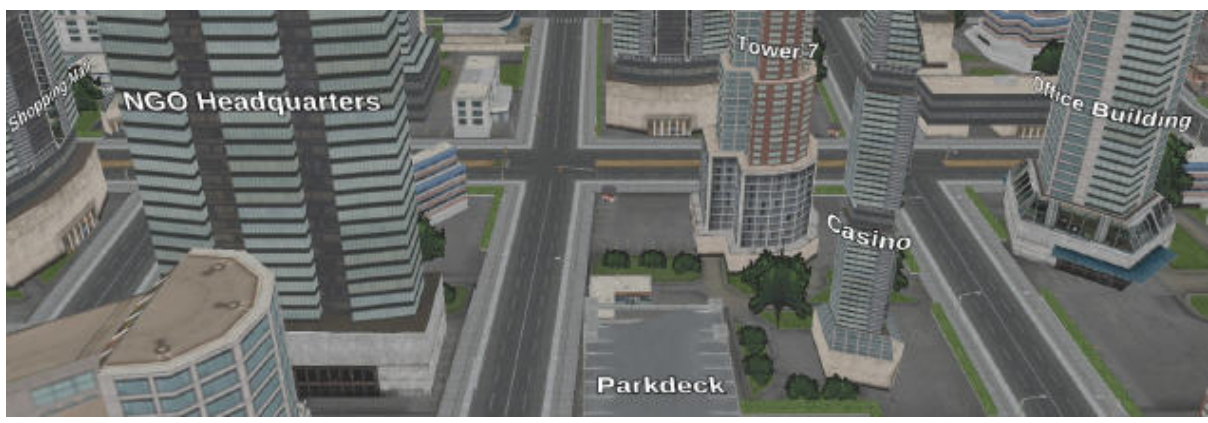

Fig. 7. 3D labels

\section{Model for the Formal Analysis of Representation Techniques}

In order to explain our model, we concentrate on a single generic task that is acquiring information about an object or a part of the scene. In what follows we consider techniques for presenting information related to a focal object, which can be geometric or non-geometric. When analyzing the usability of a display technique several factors must be taken into account:

- the number and type of information objects to display

- the semantic relations to display and the types of the related objects

- the geometric context of the focus object

It is obvious that certain techniques are not appropriate for some type of information. For instance, a 3D label [7] can only be used to display relatively short texts or icons. It is certainly inappropriate for displaying a long text document. In addition, a label placed on or close to the referenced object cannot be read if it is surrounded by large objects.

Our goal is not only to describe the usability of the techniques but also to formally specify 3D interfaces and use these specifications to automatically or semiautomatically generate the interfaces. Thus we need a formal framework to express usability of the techniques. For this purpose we will use of an information model that is close to usual object-oriented models and we will express the above-defined factors in description logics [17].

\subsection{Information Model}

The information model we consider is a classes-property model. Each object of the semantically enriched 3D scene is an instance of a class. It may be linked to other objects through properties, which are binary relations. We distinguish two main categories of classes:

Geometric classes: these are classes of objects that have a geometric representation but also non-geometric properties (e.g. a room has a geometry but it may also have a capacity, a name, a temperature, etc.). The representation of a geometric object in a scene is given either by its geometry and position or by the geometry and position of its components. 
Non-geometric (or abstract) classes: these are concepts that do not have a direct geometric representation in this environment (e.g. an emergency procedure or a building permit). Non-geometric classes include basic datatype classes (Integer, String, etc.), generic document classes such as Image, Sound, Movie, Text, or Multimedia_document, and associations. Associations (as in UML) serve to materialize semantic relations between geometric or non-geometric objects.

Display situation. A display situation is relative to a focal object and it is comprised of a display set, and a geometric context. The focal object is the geometric object is currently observing or analyzing. The display set is set of non-geometric objects that must be displayed to describe the focal object. These object may be simple data (integer, strings, ...), documents (images, sounds, ...), or semantic associations connecting the focal object to other objects. The geometric context is made of the scene objects that lie in some neighborhood of the focal object.

We propose to use description logics expressions to describe display situations. For this purpose we define the following logical vocabulary:

- the basic concepts are the classes of the information model (Geometric_object, Non_geometric_oject, Integer, String, Image, Association, ...)

- the display role is intended to connect focal object to each object of the display set

- if an object to display is an association, the target role connects it to the object(s) it associates to the focus object. An association may also bear one or more labels (connected through the label role).

- there are roles corresponding to the usual geometric and topological relations such as (above, below, within-radius- $k, \ldots$, proper-part, intersects, disjoint, ...). These roles typically link the focus object to the objects that form its geometric context.

A display situation applies to a focal object if this object belongs to the concept defined by the situation expression. For example, the following expression defines a situation where all the objects to display are strings and the focal object is not below any geometric object.

\section{S1 = forall display . String and (not exists below . Geometric_Object)}

In this second example, we specify that the display set is made of associations to geometric objects that are not contained in any other geometric object

$$
\begin{aligned}
& \mathrm{S} 2=\text { forall display } . \text { (Association and forall target (Geometric and not exists } \\
& \text { proper_part_of . Geometric_Object)) }
\end{aligned}
$$

\subsection{Description of Usability of a Technique and Technique Selection}

A usability descriptor is a 4-tuple $\langle T, A, S, U>$ meaning that technique $T$, when used to perform task $A$ in situation $S$ has usability $U$. The value $U$ would typically be obtained as the result of usability tests carried out with a panel of users.

For example, the tuple

$$
\text { UD1 = <Top3DLabel, "identify object", S1, high> }
$$

would represent the fact that the Top3DLabel (imaginary) technique has usability value high for the task "identify object" in situation S1 (defined here-above). 
Technique selection. These usability descriptors can be used to select the most appropriate display technique in a 3D virtual environment. In fact, this selection problem reduces to subsumption and instance checking problems in description logics.

Given an object $o$ in a 3DVE and a display set attached to $o$ (through a display role), a usability descriptor $\langle T, A, S, U\rangle$ applies to $o$ if $o$ is an instance of $S$, i.e. if $o$ belongs to the situation described by the DL expression $S$.

If we have a set $\mathbf{U}$ of usability descriptors (obtained through usability tests), finding appropriate display techniques for performing task $A$ on $o$ amounts to find descriptors $d=\langle T, A, S, U\rangle$ in $\mathbf{U}$ such that $d$ applies to o and the usability $U$ of $d$ is maximal. Similarly, if $V$ is a situation expression, it is possible to find the best techniques for all occurrences of $V$ by finding the descriptors $\langle T, A, S, U\rangle$ such that $V$ is subsumed by $S$ and $U$ is maximal.

Since the subsumption and instance checking problems are computable, even in very expressive description logics, this provides an effective procedure for selecting display technique in 3DVE. This selection procedure can be employed as part of a process that automatically generates $3 \mathrm{DVE}$ from specifications. It can also be integrated in an interactive 3DVE design tool to help the designer choose appropriate techniques.

\section{Conclusion and Perspectives}

This paper presents the first steps toward the creation of a specification framework for semantically enriched 3DVE. These environments are characterized by the presence of non-geometric information connected to the geometric objects of the scene.

We have shown that there already exists a range of presentation techniques for non-geometric information, but they haven't been thoroughly studied in a usability perspective. In order to carry out such a study it is necessary to define characteristic factors such as the type and quantity of information relation to display.

We have proposed to use description logic expression to formally define these factors. Since reasoning in description logics is decidable (and sometimes tractable), the usability results are directly usable to automatically select the most appropriate display technique in a given context. And thus, to generate adaptive 3D interfaces.

Our ongoing research agenda includes work to acquire usability measure for the techniques presented in this paper and to build an ontology of presentation techniques coupled with a specification system.

\section{References}

1. Ritter, F., Sonnet, H., Hartmann, K., Strothotte, T.: Illustrative shadows: integrating 3D and 2D information displays. In: Proceedings of the 8th international Conference on intelligent User interfaces IUI 2003, pp. 166-173. ACM, New York (2003)

2. Science Alberta Foundation, http: / /www . wonderville.ca/

3. The Croquet Consortium, http: / / www . opencroquet. org/

4. Kadobayashi, R., Lombardi, J., McCahill, M.P., Stearns, H., Tanaka, K., Kay, A.: 3D Model Annotation from Multiple Viewpoints for Croquet. In: Proceedings of the 4th International C5 Conference, pp. 10-15. IEEE Computer Society, Washington (2006) 
5. Interactive tour of the Grand Palais, http: / /www.grandpalais.fr/visite/fr/

6. Parallel Graphics. Cortona3D, http : / /www. cortona3d. com/try/demos . php

7. Maass, S., Döllner, J.: Seamless Integration of Labels into Interactive Virtual 3D Environments Using Parameterized Hulls. In: Proceedings of the 4th International Symposium on Computational Aesthetics in Graphics, Visualization, and Imaging, pp. 33-40. Eurographics Association, Lisbon (2008)

8. Bowman, D., Kruijff, E., LaViola, J., Mine, M., Poupyrev, I.: 3D User Interfaces: Theory and Practice. Addison Wesley, Reading (2004)

9. Dachselt, R., Hinz, M.: Three-dimensional widgets revisited - towards future standardization. In: Bowman, D., Froehlich, B., Kitamura, Y., Stuerzlinger, W. (eds.) New directions in 3D user interfaces, pp. 89-92. Shaker Verlag, Aachen (2005)

10. Bowman, D., North, C., Chen, J., Polys, N., Pyla, P., Yilmaz, U.: Information-Rich Virtual Environments: Theory, Tools, and Research Agenda. In: Proceedings of ACM Virtual Reality Software and Technology, Osaka, Japan (2003)

11. Poupyrev, I., Billinghurst, M., Weghorst, S., Ichikawa, T.: Go-Go Interaction Technique: Non-Linear Mapping for Direct Manipulation in VR. In: Proceedings of the ACM Symposium on User Interface Software and Technology (1996)

12. Parush, A., Berman, D.: Navigation and orientation in 3D user interfaces: the impact of navigation aids and landmarks. Int. J. Hum.-Comput. Stud. 61(3), 375-395 (2004)

13. Reyes-Lecuona, A., Diaz-Estrella, A.: New interaction paradigms in virtual environments. In: Electrotechnical Conference, MELECON 2006, pp. 449-452. IEEE, Los Alamitos (2006)

14. Dachselt, R., Hübner, A.: Three-dimensional menus: a survey and taxonomy. Computers and Graphics 31(1), 53-65 (2007)

15. Polys, N.F.: Display Techniques in Information-Rich Virtual Environments. Doctoral Thesis. UMI Order Number: AAI3241161. Virginia Polytechnic Institute \& State University (2006)

16. Trapp, M., Glander, T., Buchholz, H., Döllner, J.: 3D Generalization Lenses for Interactive Focus + Context Visualization of Virtual City Models. In: Proceedings of the 2008 12th international Conference information Visualisation, vol. 00, IV, pp. 356-361. IEEE Computer Society, Washington (2008)

17. Borgida, A., Brachman, R.J.: Conceptual modeling with description logics. In: Baader, F., Calvanese, D., McGuinness, D.L., Nardi, D., Patel-Schneider, P.F. (eds.) The Description Logic Handbook: theory, Implementation, and Applications, pp. 349-372. Cambridge University Press, New York (2003) 